\title{
CARACTERIZACIÓN Y SELECCIÓN DE RIZOBACTERIAS PROMOTORAS DE CRECIMIENTO EN PLÁNTULAS DE CHILE POBLANO (Capsicum annuum L.)
}

\author{
Apolinar GONZÁLEZ MANCILLA ${ }^{1}$, Juan José ALMARAZ SUÁREZ ${ }^{1 *}$, Ronald FERRERA CERRATO ${ }^{1}$, \\ María del Pilar RODRÍGUEZ GUZMÁN ${ }^{1}$, Oswaldo Rey TABOADA GAYTÁN², \\ Antonio TRINIDAD SANTOS ${ }^{1}$, Alejandro ALARCÓN ${ }^{1}$ y Ramón Ignacio ARTEAGA GARIBAY ${ }^{3}$
}

\author{
${ }^{1}$ Colegio de Postgraduados, Campus Montecillo. Carretera México-Texcoco, kilómetro 36.5, Montecillo, Tex- \\ coco, Estado de México, México, C. P. 56230 \\ ${ }^{2}$ Colegio de Postgraduados, Campus Puebla. Bulevar Forjadores de Puebla 205, Santiago Momoxpan, Municipio \\ de San Pedro Cholula, Puebla, México, C. P. 72760 \\ ${ }^{3}$ Centro Nacional de Recursos Genéticos. Instituto Nacional de Investigaciones Forestales, Agrícolas y Pecua- \\ rias. Bulevar de la Biodiversidad 400, Colonia Centro, Tepatitlán de Morelos, Jalisco, México, C. P. 47600 \\ *Autor para correspondencia: jalmaraz@hotmail.com
}

(Recibido noviembre 2015; aceptado noviembre 2016)

Palabras clave: solubilización de fosfatos, producción de auxinas, microorganismos, crecimiento de plántula, Serratia

\begin{abstract}
RESUMEN
En el cultivo de chile se usan altas dosis de fertilizantes que al no ser aprovechadas totalmente por las plantas ejercen efectos negativos en el ambiente. El uso de microorganismos benéficos puede ser una alternativa para disminuir las dosis de fertilizantes. En el presente trabajo se aislaron cepas de rizobacterias de la región de la Sierra Nevada, Puebla, México, con el objetivo de evaluar su potencial para promover el crecimiento de plántulas en chile poblano. Las cepas se caracterizaron por su capacidad de solubilizar fosfatos, fijar nitrógeno y producir auxinas. Las diez mejores cepas se evaluaron por su capacidad de promover el crecimiento en plántulas de chile poblano y finalmente éstas se identificaron a nivel molecular. Se aislaron un total de 215 cepas, de las cuales 74 fueron solubilizadoras de fosfatos que liberaron entre 10 y $881 \mu \mathrm{g} / \mathrm{mL}$ de fosfato soluble, 70 cepas tuvieron habilidad de crecer en medio libre de nitrógeno y 71 cepas produjeron entre 20 y $27 \mu \mathrm{g} / \mathrm{mL}$ de indoles. Las cepas inoculadas causaron efecto significativo en el crecimiento (Tukey, $\mathrm{p}<0.05$ ), siendo las mejores Serratia plymuthica CPPC55 y Rhizobium nepotum CPAC 35, que incrementaron el peso de la parte aérea en más de $20 \%$. Ambas cepas pueden ser usadas para aumentar la calidad de plántulas de chile poblano, lo cual podría garantizar un mejor establecimiento y sanidad de éstas en campo. Las áreas cultivadas de chile poblano son una fuente de microorganismos que pueden ser utilizados como promotores del crecimiento en especies de importancia agrícola.
\end{abstract}

Key words: phosphate solubilization, auxin production, soil microorganisms, seedling growth, Serratia

\begin{abstract}
The use of high doses of fertilizers is common in chilli pepper grown, which if are not fully utilized by plants, exerts negative effects on the environment. The use of beneficial
\end{abstract}


microorganisms can be an alternative to reduce high fertilization rates. In the present work, rhizobacteria strains from the Sierra Nevada region, Puebla, Mexico, were isolated in order to assess their potential to promote plant growth of poblano chilli seedlings. The strains were characterized by their capacity to solubilize phosphates, fix nitrogen, and produce auxins. The best 10 strains were evaluated for their ability to promote plant growth, and were finally identified at the molecular level. A total of 215 strains were isolated, from which 74 strains were phosphate solubilizers, releasing between 10 and $881 \mu \mathrm{g} / \mathrm{mL}$ of soluble phosphate, 70 strains had the ability to grow in a nitrogen-free medium, and 71 strains produced between 20 and $27 \mu \mathrm{g} / \mathrm{mL}$ of indoles. The inoculated strains caused significant effect on plant growth (Tukey, $\mathrm{p}<0.05$ ), the best being Serratia plymuthica CPPC55 and Rhizobium nepotum CPAC35, which increased the shoot weight by more than $20 \%$. Both strains can be used to increase the quality of poblano chilli seedlings, which could ensure better establishment and plant health in the field. The lands where poblano chilli crops are grown are a source of microorganisms that can be used as plant growth promoters in species of agricultural importance.

\section{INTRODUCCIÓN}

El chile (Capsicum annum L.) es uno de los cultivos de mayor importancia económica dentro de la horticultura, siendo China el principal productor mundial con más de 10.7 millones de toneladas producidas, mientras que México ocupó el segundo lugar con más 1.7 millones de toneladas (FAOSTAT 2013). Dado que el chile es un cultivo rentable, es común el uso excesivo de fertilizantes que puede superar las dosis recomendadas (Salazar-Jara y Juárez-López 2013), lo cual aumenta los costos de producción y puede generar problemas ambientales y de salud. Se estima que los cultivos sólo aprovechan entre el 20 y $50 \%$ del fertilizante aplicado (Cassman et al. 2002, Cameron et al. 2013), el resto puede perderse por lixiviación o arrastre, contaminando cuerpos de agua superficiales, que impactan negativamente a los ecosistemas acuáticos (Smith y Schindler 2009) y mantos acuíferos que pueden poner en riesgo la salud humana (Weyer et al. 2001). Existen diferentes alternativas para reducir el uso excesivo de fertilizantes, una de ellas es el uso de rizobacterias promotoras de crecimiento vegetal (RPCV).

La siembra de $C$. annum se realiza en almácigo, con el propósito de obtener plántulas de mayor sanidad y vigorosidad, que garanticen subsistir su trasplante en campo (Ruso 2004, Russo y PerkinsVeazie 2010, Puerta et al. 2012). La mejores plántulas se adquieren con el suministro adecuado de nutrimentos y control de plagas y enfermedades (Guzmán y Sánchez 2003, Gravel et al. 2012). En este proceso de producción, el uso de RPCV podría brindar un beneficio a las plántulas colonizadas con ellas y así, propiciar su establecimiento en campo con un mayor éxito.

En la región de la Sierra Nevada de Puebla, laproducción de chile poblano ha disminuido por la mala calidad de las plántulas producidas (García et al. 2011) ya que en almácigos a cielo abierto el vigor bajo es frecuente y la incidencia de plántulas enfermas llega a ser de $29 \%$, constituyendo una fuente de inóculo de fitopatógenos que los agricultores transfieren al campo durante el trasplante (González et al. 2004).

Las rizobacterias poseen varios mecanismos para promover el crecimiento de las plantas, entre los que se incluye un aumento de la movilización de nutrimentos, fijación de nitrógeno, mejoramiento en la absorción de nutrientes y control biológico de patógenos (Bais et al. 2006, De-Bashan et al. 2007, Dias et al. 2009, Hariprasad y Niranjana 2009, Altomare y Tringovska 2011). Además, sintetizan sustancias reguladoras del crecimiento como giberelinas, citoquininas y auxinas y estimulan la densidad y longitud de pelos radicales, con lo que incrementan la capacidad de absorción de agua y nutrientes, que resulta en mayor crecimiento y adaptación a condiciones de sequía, acidez y alcalinidad (Sandhya et al. 2010, Saraf et al. 2011). Algunas cepas de rizobacterias ejercen efectos antagónicos sobre otros microorganismos e inducen la resistencia sistémica en las plantas (Sharma et al. 2007).

Considerando lo anterior, el presente trabajo tuvo como objetivo aislar cepas de rizobacterias en la región de la Sierra Nevada, Puebla, México, y evaluar su potencial para promover el crecimiento de plántulas de chile poblano en invernadero. 


\section{MATERIALES Y MÉTODOS}

\section{Aislamiento y selección de cepas de rizobacterias de la rizósfera de chile poblano Colecta de suelo rizosférico}

En septiembre del 2012 se colectó suelo rizosférico de chile poblano en nueve sitios de la región de la Sierra Nevada en el estado de Puebla, México. El muestreo incluyó campos de chile poblano de tres municipios: San Matías Tlalancaleca con tres sitios, cuyas altitudes fueron desde 2414 a $2467 \mathrm{msnm}$, Chiautzingo con dos sitios de altitudes de 2404 y 2425 msnm, y Huejotzingo con cuatro sitios de altitudes que van de 2284 a 2313 msnm (Cuadro I y Fig. 1).

Se colectaron cuatro muestras por sitio, que se conservaron en hielera para su trasladado al laboratorio de Microbiología del Campus Montecillo del Colegio de Postgraduados, en Montecillo, México. En los sitios de muestreo los valores de $\mathrm{pH}$ (suelo, agua 1:2) fluctuaron entre 4.9 y 7.6. Los valores de materia orgánica (MO) fueron de 0.27 a $1.48 \%$ y de nitrógeno total (NT) de 0.01 a $.07 \%$ y de fósforo (P) de $41.1 \mathrm{a} 428.8 \mathrm{mg} / \mathrm{kg}$. La textura del suelo fue clasificado como franco arenoso en los sitios uno, tres, cuatro y cinco; franco arcillo arenoso para el sitio dos; y de arena francosa para los sitios seis, siete, ocho y nueve (Cuadro I).

\section{Aislamiento de cepas de rizobacterias}

Las muestras de suelo colectadas fueron procesadas en campana de flujo laminar en condiciones de asepsia. Se asilaron cepas de rizobacterias a través de la técnica de diluciones seriales y cuenta viable en placa. Se usaron medios de cultivo específicos: Pikovskaya para bacterias solubilizadoras de fosfatos (BSP), Rennie, para fijadoras de nitrógeno (BFN) y Luria-Bertani (LB), para bacterias productoras de auxinas (BPA). En el caso particular de BPA, una vez que se tuvo crecimiento de colonias, el $50 \%$ de ellas se sembraron por duplicado en microplacas de 96 pozos que contenían $150 \mu \mathrm{L}$ de medio líquido LB, siguiendo el mismo orden de siembra en las dos microplacas, con el fin de seleccionar colonias que producen índoles. Las microplacas se incubaron por $48 \mathrm{~h}$ y posteriormente se agregaron $150 \mu \mathrm{L}$ de reactivo Salkowski a cada pozo de una de las microplacas y se incubó en obscuridad por $30 \mathrm{~min}$, el cambio de coloración a rosa fue el indicativo de producción de indoles. De la segunda microplaca, que se dejó sin alterar, se aislaron cepas de las colonias que resultaron positivas en la producción de índoles en la primera microplaca.

En el caso de bacterias solubilizadoras de fosfatos, se aislaron cepas con base en las colonias que presentaron un halo alrededor de ellas, las cuales se preservaron en agar nutritivo hasta su evaluación. Las cepas fijadoras de nitrógeno se aislaron de colonias de bacterias que tuvieron un mejor crecimiento en el medio de Rennie.

\section{Caracterización de las rizobacterias por atributos promotores del crecimiento vegetal}

Las cepas solubilizadoras de fosfato aisladas se cultivaron en medio líquido Pikovskaya (Pikovskaya 1948) por siete días en agitación. Se llenaron tubos Eppendorf con $1 \mathrm{~mL}$ de cultivo bacteriano y se centrifugaron

CUADRO I. SITIOS DE AISLAMIENTO DE LAS CEPAS DE RIZOBACTERIAS Y CARACTERÍSTICAS DEL SUELO EN EL ESTADO DE PUEBLA, MÉXICO

\begin{tabular}{|c|c|c|c|c|c|c|c|}
\hline \multirow{2}{*}{$\begin{array}{l}\text { Municipio } \\
\text { y sitios }\end{array}$} & \multirow[t]{2}{*}{ Localización } & \multirow{2}{*}{$\begin{array}{l}\text { Altitud } \\
\text { (msnm) }\end{array}$} & \multicolumn{5}{|c|}{ Características del suelo } \\
\hline & & & $\mathrm{pH}$ & MO & NT & $\mathrm{P}$ & Textura \\
\hline \multicolumn{8}{|c|}{ San Matías Tlalancaleca } \\
\hline 1 & $19^{\circ} 22.313^{\prime} \mathrm{N}$ y $98^{\circ} 32.032^{\prime} \mathrm{W}$ & 2467 & 5.5 & 0.27 & 0.01 & 184.7 & Franco arenoso \\
\hline 2 & $19^{\circ} 22.257^{\prime} \mathrm{N}$ y $98^{\circ} 31.924^{\prime} \mathrm{W}$ & 2453 & 5.8 & 0.54 & 0.03 & 224.0 & Franco arcillo arenoso \\
\hline 3 & $19^{\circ} 20.665^{\prime} \mathrm{N}$ y $98^{\circ} 29.209^{\prime} \mathrm{W}$ & 2414 & 6.8 & 1.48 & 0.07 & 314.5 & Franco arenoso \\
\hline \multicolumn{8}{|c|}{ Chiautzingo } \\
\hline 4 & $19^{\circ} 13.440^{\prime} \mathrm{N}$ y $98^{\circ} 28.903^{\prime} \mathrm{W}$ & 2425 & 6.9 & 0.94 & 0.05 & 153.7 & Franco arenoso \\
\hline 5 & $19^{\circ} 13.052^{\prime} \mathrm{N}$ y $98^{\circ} 28.261^{\prime} \mathrm{W}$ & 2404 & 4.9 & 0.27 & 0.01 & 187.1 & Franco arenoso \\
\hline \multicolumn{8}{|c|}{ Huejotzingo } \\
\hline 6 & $19^{\circ} 08.504^{\prime} \mathrm{N}$ y $98^{\circ} 24.700^{\prime} \mathrm{W}$ & 2313 & 5.8 & 0.40 & 0.02 & 41.5 & Arena francosa \\
\hline 7 & $19^{\circ} 08.968^{\prime} \mathrm{N}$ y $98^{\circ} 24.430^{\prime} \mathrm{W}$ & 2292 & 6.7 & 0.94 & 0.05 & 428.8 & Arena francosa \\
\hline 8 & $19^{\circ} 11.985^{\prime} \mathrm{N}$ y $98^{\circ} 25.711^{\prime} \mathrm{W}$ & 2286 & 7.3 & 1.48 & 0.07 & 50.1 & Arena francosa \\
\hline 9 & $19^{\circ} 12.295^{\prime} \mathrm{N}$ y $98^{\circ} 25.510^{\prime} \mathrm{W}$ & 2284 & 7.6 & 0.67 & 0.03 & 44.3 & Arena francosa \\
\hline
\end{tabular}

$\mathrm{MO}=$ materia orgánica $(\%), \mathrm{NT}=$ nitrógeno total $(\%), \mathrm{P}=$ fósforo $(\mathrm{mg} / \mathrm{kg})$ 


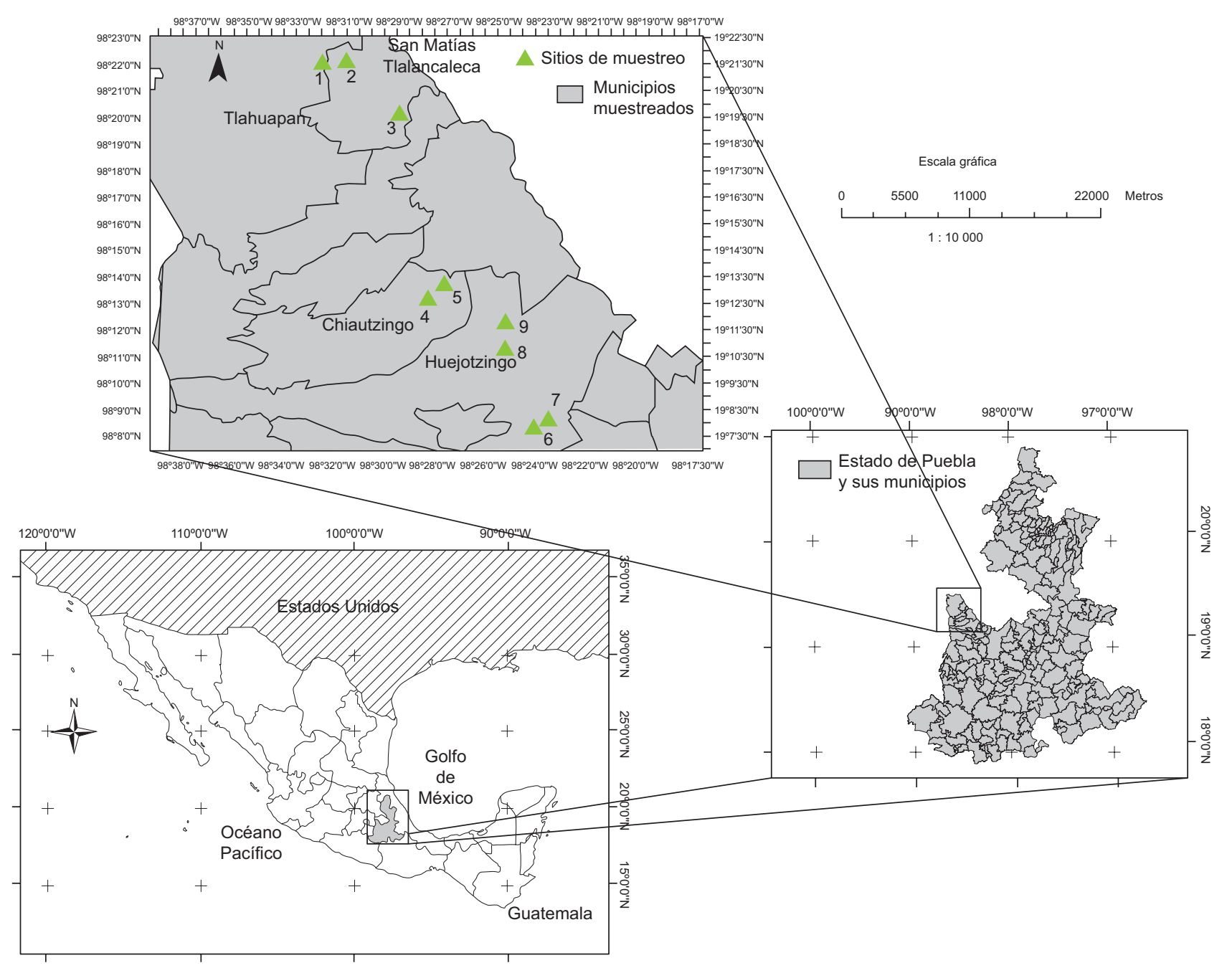

Fig. 1. Ubicación geográfica de los nueve sitios donde se colectaron muestras de suelo rizosférico de chile poblano en la Sierra Nevada, estado de Puebla, México

a $5000 \mathrm{rpm}$ durante $20 \mathrm{~min}$. Del sobrenadante obtenido de cada tubo se tomaron alícuotas de $200 \mu \mathrm{L}$ y se colocaron en microplacas de poliestireno marca Costar (Corning Incorporated, NY), después se les adicionaron $100 \mu \mathrm{L}$ de solución de vanadato $\left(\mathrm{NH}_{4} \mathrm{VO}_{3} 0.25 \%\right.$ en $\left.35 \% \mathrm{HNO}_{3}\right)$ y $100 \mu \mathrm{L}$ de solución de molibdato $\left[\left(\mathrm{NH}_{4}\right)_{6} \mathrm{MO}_{7} \mathrm{O}_{24}\right.$ en $5 \%$ en agua]. Las muestras se dejaron en reposo durante 5 min hasta que desarrollaron color y posteriormente se realizaron las lecturas en el espectrofotómetro a $420 \mathrm{~nm}$. Los cálculos para la determinación de fosfato soluble se realizaron a partir de una curva estándar, la cual se elaboró con diferentes concentraciones de fosfato $(0,20,40,60,100,150,200,250$ y $300 \mu \mathrm{g} / \mathrm{mL})$, que fueron procesadas de la misma forma que los extractos bacterianos.

Las cepas productoras de índoles fueron sembradas en medio de cultivo líquido Luria-Bertani y se mantuvieron en agitación durante cuatro días. Posteriormente se pasó $1 \mathrm{~mL}$ de cada muestra a tubos Eppendorf y se centrifugó por 20 min a 5000 $\mathrm{rpm}$. Del sobrenadante se tomaron alícuotas de 150 $\mu \mathrm{L}$ y fueron colocadas en microplacas, a las cuales se les adicionaron $100 \mu \mathrm{L}$ de reactivo Salkowski (2 $\% 0.5 \mathrm{M} \mathrm{FeCl}_{3}$ en $35 \%$ de ácido perclórico). Las muestras fueron incubadas por $30 \mathrm{~min}$ en oscuridad y después se procedió a tomar las lecturas en el espectrofotómetro a una absorbancia de $530 \mathrm{~nm}$. La producción de indoles se cuantificó mediante una curva estándar usando diferentes concentraciones de ácido indolacético que fueron de $0,10,15,20,25$, 30,40 y $50 \mu \mathrm{g} / \mathrm{mL}$. En el caso de las cepas fijadoras de nitrógeno la evaluación sólo fue cualitativa, esto es, se seleccionaron exclusivamente por su capacidad de crecer en el medio de Rennie, un medio libre de nitrógeno. 


\section{Identificación molecular}

Se extrajo el material genético de biomasa equivalente a siete colonias de las cepas a partir de cultivos jóvenes de $24 \mathrm{~h}$ mediante el equipo comercial EZ-10 Spin ${ }^{\circledR}$ (Column Bacterial DNA mini-preps kit, Bio Basic Inc., Canadá). Se realizó la amplificación de un fragmento de $\approx 1500$ pb del gen $16 \mathrm{~S}$ $\mathrm{ADNr}$ para realizar la asignación de identidad de manera confiable hasta nivel de género y especie. Los iniciadores empleados para la amplificación del gen 16S ADNr fueron el 27f (forward) 5'-AGA GTT TGA TCM TGG CTC AG-3' y el 1492r (reverse) 5'-CGG TTA CCT TGT TAC GAC TT-3' (Frank et al. 2008).

La mezcla de reacción de amplificación fue con $1 \mathrm{X}$ de regulador de la reacción en cadena de la polimerasa (PCR), $2 \mu \mathrm{M}$ de desoxinucleósidos trifosfatados (dNTP's), $0.2 \mu \mathrm{M}$ de $\mathrm{MgCl}_{2}, 10 \mu \mathrm{M}$ de cada iniciador y $0.05 \mathrm{U} / \mu \mathrm{L}$, en un volumen final de $25 \mu \mathrm{L}$. Se utilizó el programa de termociclador con una desnaturalización inicial de 5 min a $95^{\circ} \mathrm{C}, 30$ ciclos de: desnaturalización por 1 min a $94{ }^{\circ} \mathrm{C}$, alineación de 1 min a $57^{\circ} \mathrm{C}$, y extensión de 1.5 min a $72{ }^{\circ} \mathrm{C}$, se utilizó una extensión final a $72{ }^{\circ} \mathrm{C}$ por $7 \mathrm{~min}$.

Los amplicones fueron visualizados en geles de agarosa al $1.5 \% \mathrm{p} / \mathrm{v}$, se tiñeron con colorante verde de ADN (Green-DNA dye, Bio Basic Inc., Canadá) para visualizar en un transiluminador. Se purificaron los productos de PCR con el sistema comercial de purificación Agencourt ${ }^{\circledR}$ AMPure ${ }^{\circledR}$ XP (Beckman Coulter, EUA), se siguieron las indicaciones de uso del fabricante. Los amplicones purificados fueron enviados al servicio de secuenciación Macrogen, Corea.

Las secuencias fueron editadas con los programas BioEdit 7.0.9.0 (Hall 1999) y Seaview 4.0
(Galtier et al. 1996). Se efectuó un análisis en la plataforma Blast (http://blast.ncbi.nlm.nih.gov/ Blast.cgi) para la búsqueda de genes homólogos a las secuencias, considerándose los criterios de Rosselló-Mora y Amann (2001) para la asignación a nivel de especie con porcentajes de identidad superiores al $97 \%$ y de género al 95-96\%, con cobertura mayor al $85 \%$. Se hizo la filogenia en el programa Mega versión 6.0 (Tamura et al. 2013) con una minería de secuencias del GenBank (http:// www.ncbi.nlm.nih.gov/genbank/) obtenidas del análisis MegaBlast (Morgulis et al. 2008) para corroborar la identidad mediante la concordancia de la topología y la identidad del Blast para cada una de las secuencias de las cepas.

\section{Efectividad de las rizobacterias en la promoción del crecimiento en plántulas de chile poblano Preparación del inóculo}

Se incluyeron diez cepas de bacterias para evaluar sus efectos en la promoción de crecimiento, las cuales fueron seleccionadas en el laboratorio por su capacidad para solubilizar fosfatos, producir auxinas o fijar nitrógeno. Las cepas bacterianas se cultivaron en caldo nutritivo en agitación constante por tres días a $28^{\circ} \mathrm{C}$ hasta obtener una concentración de $10^{9}$ células $/ \mathrm{mL}$. Se compararon con dos testigos: uno sin inocular y otro que consistió en la inoculación de la cepa bacteriana Bacillus pumillus R44 con probada capacidad para promover crecimiento, la cual fue proporcionada por el Laboratorio de Microbiología del Colegio de Postgraduados. Cuatro de las diez cepas utilizadas poseen capacidad para solubilizar fosfatos, cuatro más producen auxinas y dos son fijadoras de nitrógeno (Cuadro II).

CUADRO II. AFILIACIÓN GENÉTICA DE LAS CEPAS DE ESTUDIO SEGÚN LAS SECUENCIAS DE AMPLIFICACIÓN DEL GEN 16S ADNr COMPARADAS CON EL GENBANK

\begin{tabular}{lllcccc}
\hline $\begin{array}{l}\text { Clave de } \\
\text { identificación }\end{array}$ & Característica & Identificado como & $\begin{array}{c}\text { Pares de } \\
\text { bases }\end{array}$ & $\begin{array}{c}\text { Número de acceso } \\
\text { al GenBank }\end{array}$ & $\begin{array}{c}\text { Cobertura } \\
(\%)\end{array}$ & $\begin{array}{c}\text { Similitud } \\
(\%)\end{array}$ \\
\hline CPPC6 & BSP & Serratia plymuthica & 899 & KX259562 & 100 & 100 \\
CPPC22 & BSP & Serratia marcescens & 899 & KX259561 & 100 & 99 \\
CPPC54 & BSP & Serratia plymuthica & 905 & KX259563 & 100 & 99 \\
CPPC55 & BSP & Serratia plymuthica & 903 & KX259564 & 100 & 99 \\
CPAC20 & BPA & Bacillus sp. & 901 & KX258421 & 100 & 99 \\
CPAC35 & BPA & Rhizobium nepotum & 902 & KX258419 & 100 & 99 \\
CPAC36 & BPA & Arthrobacter sp. & 900 & KX258420 & 100 & 99 \\
CPAC53 & BPA & Serratia liquefaciens & 1544 & KX259559 & 100 & 100 \\
CPNC9 & BFN & Stenotrophomonas maltophilia & 898 & KX259565 & 100 & 99 \\
CPNC10 & BFN & Serratia marcescens & 621 & KX259560 & 100 & 99 \\
\hline
\end{tabular}

$\mathrm{BSP}=$ bacterias solubilizadoras de fosfatos, $\mathrm{BPA}=$ bacterias productoras de auxinas, $\mathrm{BFN}=$ bacterias fijadoras de nitrógeno, $\mathrm{CPPC}=$ Colegio de Postgraduados, solubilizadoras de fosfatos en Capsicum, $\mathrm{CPAC}=$ Colegio de Postgraduados, productoras de auxinas en Capsicum, CPNC = Colegio de Postgraduados, fijadoras de nitrógeno en Capsicum 


\section{Establecimiento del experimento}

El sustrato utilizado para el crecimiento de las plántulas de chile poblano fue una mezcla de musgo de turba (peat moss), perlita y vermiculita (relación $1: 1: 1)$, mismo que fue esterilizado en autoclave a $18 \mathrm{lb}$ de presión por $3 \mathrm{~h}$. Con el sustrato se llenaron semilleros de 20 cavidades y después se sembraron dos semillas por cada cavidad. Antes de la siembra, la semilla de chile fue desinfectada con cloro al 1 $\%$ por 3 min y lavada tres veces con agua destilada estéril. A la emergencia se dejó una plántula por cavidad y se inoculó $1 \mathrm{~mL}$ de suspensión bacteriana. A los 68 días después de la inoculación las plántulas fueron cosechadas. Se midió altura con una regla y el área foliar se determinó con un medidor de área foliar (Area Meter, Modelo LI-3100; Nebraska, EUA). La parte aérea y la raíz se secaron hasta peso constante en un horno (Felisa, Modelo 242-A, Ciudad de México México) a $70{ }^{\circ} \mathrm{C}$ por $72 \mathrm{~h}$; La biomasa seca se determinó en una balanza analítica (Sartorius, Modelo Analytic AC 210S, Illinois, EUA). El experimento fue establecido utilizando un diseño experimental completamente al azar con 12 tratamientos (incluyendo los testigos) y 20 repeticiones. Los datos obtenidos fueron analizados mediante el paquete estadístico SAS para Windows (SAS Institute Inc. 2002), realizando un análisis de varianza y prueba de comparación de medias (Tukey, $\alpha=0.05$ ).

\section{RESULTADOS Y DISCUSION}

\section{Aislamiento y caracterización de rizobacterias por sus atributos de promoción del crecimiento}

Se aislaron un total de 215 cepas, de las cuales 74 fueron solubilizadoras de fosfatos, 70 fijadoras de nitrógeno y 71 cepas fueron productoras de indoles. Las cepas fijadoras de nitrógeno sólo se seleccionaron con base en su capacidad de crecer en medio de Rennie, el cual es un medio libre de nitrógeno. En el caso de los otros dos grupos de cepas se realizó una evaluación cuantitativa.

En las 74 cepas solubilizadoras de fosfato aisladas se encontró que éstas liberaron entre 10 y $881 \mu \mathrm{g} / \mathrm{mL}$ de fosfato cuando se cultivaron en medio líquido. El $31 \%$ de las cepas solubilizaron más de $400 \mu \mathrm{g} / \mathrm{mL}$ de fosfato, de estas, seis cepas (CPPC23, CPPC24, CPPC25, CPPC26, CPPC28, CPPC29) provienen del sitio 3 de San Matías Tlalancaleca a $2414 \mathrm{msnm}$ (Cuadro I). La mejor fue la cepa CPPC25 con $881 \mu \mathrm{g} / \mathrm{mL}$ de fosfato solubilizado (Fig. 2). Varios estudios han evidenciado que diversos géneros de bacterias poseen la capacidad para solubilizar fosfatos, destacando Pseudomonas, Bacillus, Rhizobium, Burkholderia, Achromobacter, Microccocus, Aereobacter, Flavobacterium, Enterobacter, Pantotea, Klebsiella, Rhodobacter, Arthrobacter, Serratia y Erwinia (Khan et al. 2010, Datta et al. 2011), los cuales han sido utilizados en plantas de arroz (Chaiharn y Lumyong 2009), trigo (Ahmad et al. 2008), soya (Son et al. 2006), maíz (Fitriatin et al. 2014), tomate (Banerjee et al. 2010) y chile (Datta et al. 2011, Hahm et al. 2012, Luna et al. 2013, ReyesRamírez et al. 2014).

En el caso de la producción de ácido indolacético, se realizó una preselección de 71 cepas con base en el cambio de color a rosa cuando al cultivo bacteriano se le adicionó el reactivo de Salkowski (indicativo de producción de indoles). La evaluación cuantitativa indicó que estas cepas produjeron entre 21 y $26 \mu \mathrm{g} / \mathrm{mL}$ de índoles, de las cuales el $17 \%$ fueron aisladas en el sitio 3 de San Matías Tlalancaleca a $2414 \mathrm{msnm}$, el $15 \%$ fueron del sitio 7 de Huejotzingo a $2292 \mathrm{msnm}$, y el $13 \%$ de los sitios 4 (Chiautzingo, a $2425 \mathrm{msnm}$ ) y 9 (Huejotzingo, a $2284 \mathrm{msnm}$ ). La cantidad más alta de indoles producidos fue encontrada en la cepa CPAC52, proveniente del sitio 7 del municipio de Huejotzingo con $26.4 \mu \mathrm{g} / \mathrm{mL}$ (Cuadro I y Fig. 3). La producción de auxinas por parte de las cepas está condicionada por el tipo y edad del cultivo asociado, además de la especie y género al cual pertenece la bacteria (Ponmurugan y Gopi 2006).

La solubilización de fosfatos por las cepas evaluadas en este estudio fue alta respecto a lo encontrado por Datta et al. (2011), quienes al evaluar cepas de rizobacterias aisladas en rizósfera de $C$. annuum cultivar Suryamukhi encontraron que el nivel más alto de fosfato solubilizado $(55 \mu \mathrm{g} / \mathrm{mL})$ lo presentó la cepa Streptomyces sp. C32, el ácido indol acético producido por esta cepa fue menos de $10 \mu \mathrm{g} / \mathrm{mL}$. En otro estudio, Arthrobacter sp. y Bacillus sp., aislados en rizósfera de tomate tuvieron valores altos de solubilización de fosfatos, que fueron entre 150 y 200 $\mu \mathrm{g} / \mathrm{mL}$ (Banerjee et al. 2010).

\section{Identificación molecular de las cepas}

Las diez mejores cepas en sus atributos de promoción de crecimiento se identificaron a nivel molecular. Seis cepas identificadas pertenecen al género Serratia. De éstas, tres fueron de la especie Serratia plymuthica (CPPC6, CPPC54 y CPPC55), dos cepas pertenecen a Serratia mascescens (CPPC22 y CPNC10) y una fue de la especie Serratia liquefaciens (CPAC53). Las cepas CPAC35, CPAC36, 

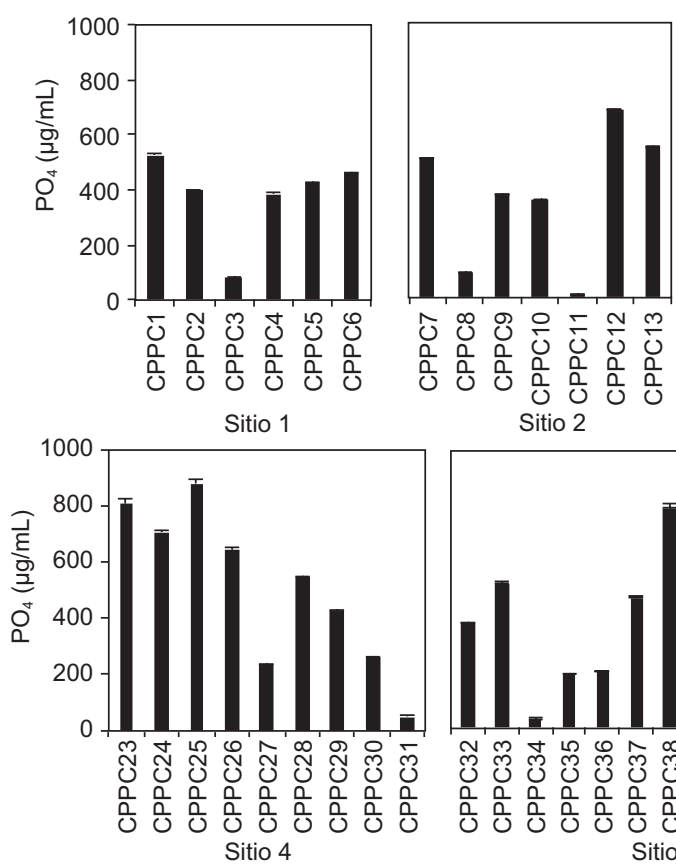

Sitio 2
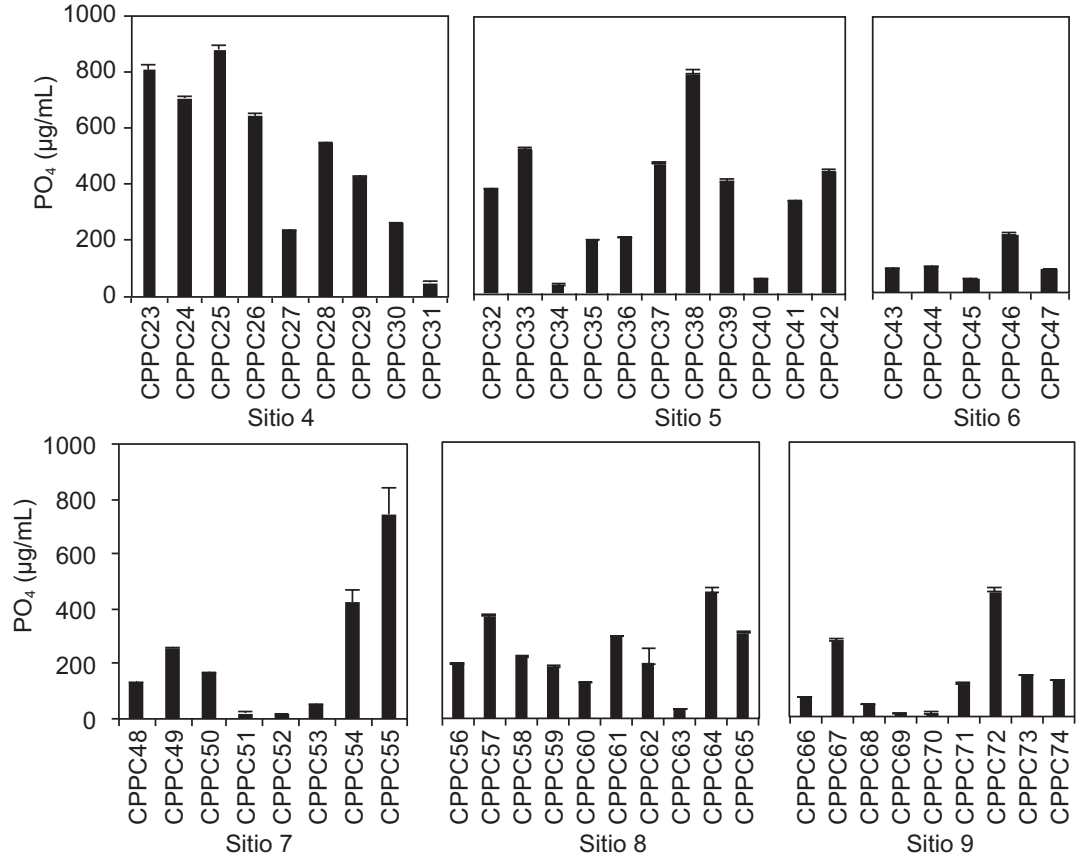

Fig. 2. Solubilización de fosfatos $\left(\mathrm{PO}_{4}\right)$ por las cepas de bacterias solubilizadoras de fosfatos aislados en suelo rizosférico de chile poblano colectado en nueve sitios de la Sierra Nevada, estado de Puebla, México. Medias $n=3, \pm$ error estándar. $\mathrm{CPPC}=$ Colegio de Postgraduados, solubilizadoras de fosfatos en Capsicum

CPAC20 y CPNC9 pertenecen a Rhizobium nepotum, Arthrobacter sp., Bacillus sp. y Stenotrophomonas maltophilia, respectivamente (Cuadro II).

Efectividad de las rizobacterias en la promoción del crecimiento en plántulas de chile poblano

Las cepas bacterianas inoculadas en plántulas de chile mostraron diferencias estadísticas significativas (Tukey, $\mathrm{p} \leq 0.05$ ). Los resultados evidenciaron que el $49 \%$ de los tratamientos inoculados con las rizobacterias mostraron incrementos en altura, área foliar y producción de biomasa seca total respecto al testigo. Los mejores tratamientos fueron las plántulas inoculadas con Rhizobium nepotum CPAC35 y Serratia plymuthica CPPC55. El tratamiento con la cepa CPA35 obtuvo una altura final promedio de $9.6 \mathrm{~cm}$, $12 \mathrm{~cm}^{2}$ de área foliar y $82 \mathrm{mg}$ de biomasa seca total, valores que fueron $22.5,7.6$ y $26.04 \%$ mayores en comparación con el testigo, respectivamente. El tratamiento con la cepa CPPC55 incrementó altura $(9 \mathrm{~cm})$ en $15 \%$, área foliar $\left(13 \mathrm{~cm}^{2}\right)$ en $34 \%$ y producción de biomasa seca $(72 \mathrm{mg})$ en $11 \%$ (Cuadro III).

En un estudio similar Yau et al. (2013) encontraron incrementos en altura, diámetro, número de hojas, biomasa seca y área foliar por efecto de la inoculación de $S$. marcescens y Bacillus cereus en plantas de chile, debido a que estos microorganismos tienen la capacidad de producir acido indolacético e inhibir fitopatógenos como $P$. capsicí hasta en un $75 \%$. En otros trabajos se han encontrado efectos benéficos al inocular $S$. liquefaciens en cultivos de trigo (Abaid-Ullah et al. 2015), tomate (Manas et al. 2010), soya (Pan et al. 2002) y maíz (Pan et al. 1999), pero 


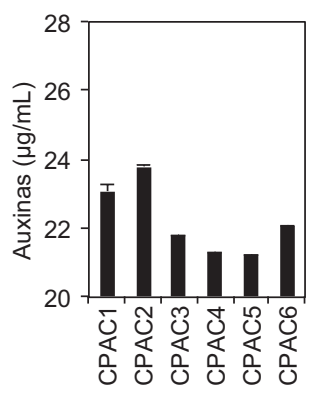

Sitio 1
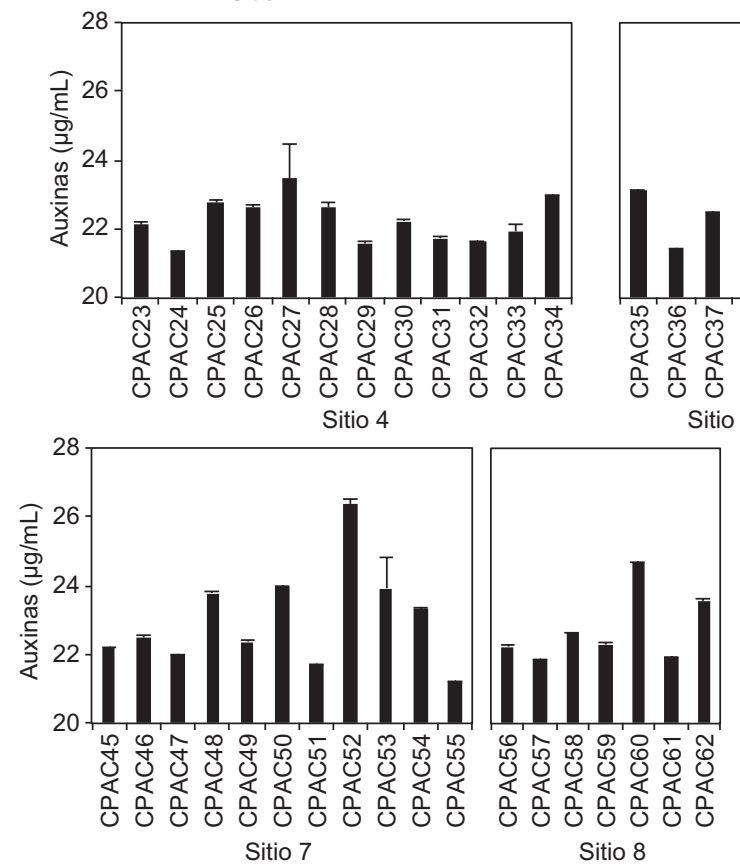

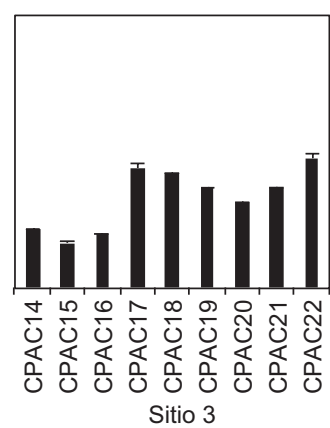

Sitio 3
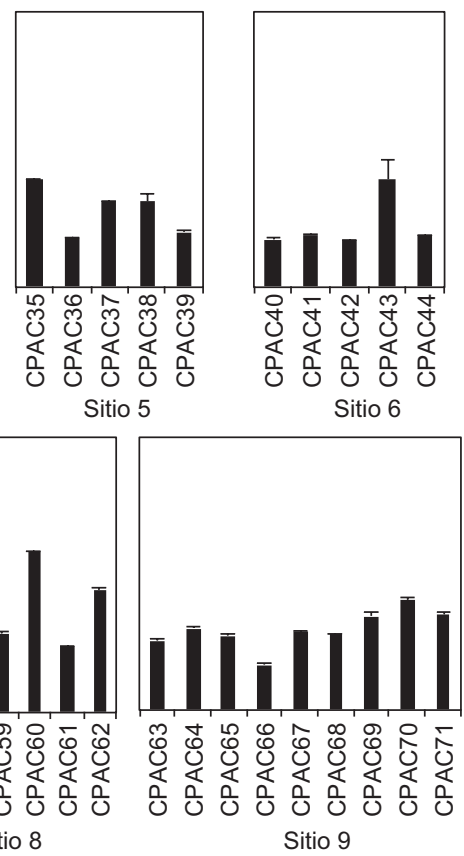

Fig. 3. Producción de auxinas por las cepas de bacterias productoras de auxinas aislados en suelo rizosférico de chile poblano colectado en nueve sitios de la Sierra Nevada, estado de Puebla, México. Medias $n=3, \pm$ error estándar. $\mathrm{CPAC}=$ Colegio de Postgraduados, productoras de auxinas en Capsicum

no se han encontrado reportes en cultivos de chile, siendo este uno de los primeros estudios en cuanto a la calidad de plántulas de chile poblano.

Serratia plymuthica es una bacteria ubicua Gram negativa que se ha aislado en rizósfera de plantas de todo el mundo, ya sea en vida libre o como organismo endófito (De Vleesschauwer y Hofte 2007) y tiene potencial no sólo para suprimir los agentes patógenos del suelo, sino también para estimular el crecimiento vegetal mediante la exudación de sideróforos, producción de acido indol acético, creación de películas protectoras de la rizósfera y biosíntesis de quitinasas y proteasas (Muller et al. 2009). Sus efectos benéficos como rizobacteria promotora del crecimiento han sido evaluados en plantas de papa (Czajkowski et al. 2012), pepino (McCullagh et al. 1996), algodón, frijol, manzana, arroz, naranja, lechuga, fresa (De Vleesschauwer y Hofte 2007) y chile (Shen et al. 2006, Kim et al. 2008, Cao et al. 2009), encontrándose incrementos en altura, biomasa radical y foliar y rendimiento de fruto en el cultivo. Específicamente en C. annuum, Kim et al. (2008) reportaron que esta rizobacteria tiene la capacidad de disminuir la incidencia de patógenos como $P$. capsici, Rhizoctonia solani, Fusarium oxysporum y $F$. solani.

En otros trabajos también se han encontrado incrementos en la calidad de plántulas de $C$. annuum (Datta et al. 2011, Hahm et al. 2012, Luna et al. 2013) y C. chinense Jacq. (Reyes-Ramírez et al. 2014) al evaluar parámetros como germinación, altura, diámetro, biomasa seca, área foliar y rendimiento mediante la inoculación de rizobacterias. A pesar de los efectos positivos encontrados en este trabajo al inocular Rhizobium nepotum CPAC35 en plántulas 
CUADRO III. ALTURA, ÁREAFOLIAR Y BIOMASA SECA EN PLÁNTULAS DE CHILE POBLANO POR EFECTO DE LA INOCULACIÓN DE RIZOBACTERIAS AISLADAS DE LA RIZÓSFERA DE CHILE POBLANO A LOS 68 DÍAS DESPUÉS DE LA INOCULACIÓN

\begin{tabular}{lcccc}
\hline Cepas & $\begin{array}{c}\text { Altura final } \\
(\mathrm{cm})\end{array}$ & $\begin{array}{c}\text { Área foliar } \\
\left(\mathrm{cm}^{2}\right)\end{array}$ & \multicolumn{2}{c}{ Biomasa seca $(\mathrm{mg})$} \\
\cline { 4 - 5 } & & & Radical & Aérea \\
\hline Testigo & $7.8 \mathrm{cde}$ & $10 \mathrm{de}$ & $23 \mathrm{bc}$ & $42 \mathrm{bc}$ \\
R44 & $8.5 \mathrm{bc}$ & $11 \mathrm{bcd}$ & $20 \mathrm{cde}$ & $46 \mathrm{ab}$ \\
CPPC6 & $7.9 \mathrm{cde}$ & $11 \mathrm{bcd}$ & $23 \mathrm{bc}$ & $41 \mathrm{bcd}$ \\
CPPC22 & $7.7 \mathrm{cde}$ & $10 \mathrm{~cd}$ & $15 \mathrm{f}$ & $36 \mathrm{cde}$ \\
CPPC54 & $8.1 \mathrm{bcd}$ & $11 \mathrm{bcd}$ & $18 \mathrm{def}$ & $41 \mathrm{bcd}$ \\
CPPC55 & $9.0 \mathrm{ab}$ & $13 \mathrm{a}$ & $21 \mathrm{cde}$ & $51 \mathrm{a}$ \\
CPAC20 & $7.2 \mathrm{de}$ & $8 \mathrm{e}$ & $22 \mathrm{~cd}$ & $34 \mathrm{de}$ \\
CPAC35 & $9.6 \mathrm{a}$ & $12 \mathrm{ab}$ & $30 \mathrm{a}$ & $53 \mathrm{a}$ \\
CPAC36 & $7.5 \mathrm{cde}$ & $10 \mathrm{~cd}$ & $19 \mathrm{def}$ & $41 \mathrm{bcd}$ \\
CPAC53 & $7.8 \mathrm{cde}$ & $11 \mathrm{abc}$ & $23 \mathrm{bc}$ & $42 \mathrm{bc}$ \\
CPNC9 & $6.9 \mathrm{e}$ & $9 \mathrm{de}$ & $17 \mathrm{ef}$ & $33 \mathrm{e}$ \\
CPNC10 & $7.4 \mathrm{de}$ & $11 \mathrm{abcd}$ & $27 \mathrm{ab}$ & $40 \mathrm{bcde}$ \\
\hline
\end{tabular}

Letras distintas dentro de la misma columna, denotan diferencias estadísticas significativas (Tukey, $\alpha=0.05, \mathrm{a}>\mathrm{b}$ ). CPPC $=$ Colegio de Postgraduados, solubilizadoras de fosfatos en Capsicum, $\mathrm{CPAC}=$ Colegio de Postgraduados, productoras de auxinas en Capsicum, $\mathrm{CPNC}=$ Colegio de Postgraduados, fijadoras de nitrógeno en Capsicum

de chile poblano, Pulawska et al. (2012) mencionan que este género de bacterias podría estar asociado a la formación de agallas radicales, principalmente en algunas especies de frutales como el ciruelo (Prunus cerasifera), la frambruesa (Rubus idaeus) y el cerezo (Prunus avium L.). Sin embargo, en esta investigación lo que se observó fue un incremento en el crecimiento de las plántulas.

En este trabajo se demostró que la inoculación de rizobacterias promueve crecimiento de plántulas de chile poblano, con lo que se obtienen ejemplares de mayor calidad. Probablemente los beneficios al obtener mayor altura, área foliar y biomasa seca en las plántulas son una consecuencia de la producción de ácido indolacético (la CPAC35 produjo $23 \mu \mathrm{g} / \mathrm{mL}$ ) y solubilización de fosfatos (la CPPC55 liberó $744 \mu \mathrm{g}$ / $\mathrm{mL}$ ), lo cual también fue observado por Sarathambal et al. (2015). Además de estas características, posiblemente las cepas tengan otras propiedades que necesitan ser evaluadas y que no fueron estudiadas en este trabajo, por ejemplo producción de sideroforos y de antifúngicos. En un estudio Amaresan et al. (2012) encontraron que la inoculación de Bacillus sp., Serratia sp., Arthrobacter sp. y Serratia marcescens incrementan la calidad de plántulas de tomate (Lycopersicon esculentum) y chile (C. annuum) al haber aumentos en la longitud radical y numero de raíces secundarias, mayor altura y producción de biomasa seca.

\section{CONCLUSIONES}

El uso de rizobacterias promotoras de crecimiento con capacidad para solubilizar fosfatos y producir auxinas es efectivo para obtener plántulas de buena calidad en chile poblano. Serratia plymuthica CPPC55 y Rhizobium nepotum CPAC35 promovieron mayor altura y mejor producción de biomasa seca radical y área en plántulas de chile poblano. El uso de microorganismos benéficos son alternativas para disminuir el uso de fertilizantes sintéticos y reducir sus efectos adversos en el ambiente y en la salud humana. Serratia liquefaciens CPAC53 no se ha utilizado como promotor de crecimiento en Capsicum, a pesar de no encontrar efectos positivos en altura se incrementó el área foliar de las plántulas, por lo que esta cepa al igual que Serratia plymuthica CPPC55 podrían ser usadas para la obtención de plántulas con la calidad necesaria al momento del transplante y que podrían favorecer el rendimiento del chile poblano.

\section{REFERENCIAS}

Abaid-Ullah M., Hassan M. N., Jamil M., Brader G., Shah M. K. N., Sessitsch A. y Hafeez F. Y. (2015). Plant growth promoting rhizobacteria: An alternate way to improve yield and quality of wheat (Triticum aestivum). Int. J. Agric. Biol. 17 (1), 51-60.

Ahmad F., Ahmad I. y Khan M. S. (2008). Screening of free-living rhizobacteria for their multiple plant growth promoting activities. Microbiol. Res. 163 (2), 173-181. DOI: $10.1016 /$ j.micres.2006.04.001

Altomare C. y Tringovska I. (2011). Beneficial soil microorganisms, an ecological alternative for soil fertility management. En: Genetics, biofuels and local farming systems (E. Lichtfouse, Ed.). Sustainable agriculture reviews, Vol. 7. Springer, Países Bajos. pp. 161-214. DOI: 10.1007/978-94-007-1521-9_6

Amaresan N., Jayakumar V., Kumar K. y Thajuddin N. (2012). Isolation and characterization of plant growth promoting endophytic bacteria and their effect on tomato (Lycopersicon esculentum) and chilli (Capsicum апnиит) seedling growth. Ann. Microbiol. 62 (2), 805-810. DOI: $10.1007 / \mathrm{s} 13213-011-0321-7$

Bais H. P., Weir T. L., Perry L. G., Gilroy S. y Vivanco J. M. (2006). The role of root exudates in rhizosphere 
interactions with plants and other organisms. Annu. Rev. Plant Biol. 57, 233-66.

DOI: 10.1146/annurev.arplant.57.032905.105159

Banerjee S., Palit R., Sengupta C. y Standing D. (2010). Stress induced phosphate solubilization by Arthrobacter sp. and Bacillus sp. isolated from tomato rhizosphere. AJCS 4 (6), 378-383.

Cameron K. C., Di H. J. y Moir J. L. (2013). Nitrogen losses from the soil/plant system: a review. Ann. Appl. Biol.162 (2), 145-173.

DOI: $10.1111 / \mathrm{aab} .12014$

Cao P., Shen S. S., Wen C. Y., Song S. y Park C. S. (2009). The effect of the colonization of Serratia plymuthica A21-4 in rhizosphere soil and root of pepper in different soil environment. Res. Plant Dis. 15 (2), 101-105. DOI: 10.5423/RPD.2009.15.2.101

Cassman K. G., Dobermann A. R. y Walters D. T. (2002). Agroecosystems, nitrogen-use efficiency, and nitrogen management. Ambio 31 (2), 132-140.

DOI: $10.1579 / 0044-7447-31.2 .132$

Chaiharn M. y Lumyong S. (2009). Phosphate solubilization potential and stress tolerance of rhizobacteria from rice soil in Northern Thailand. World J. Microbiol. Biotechnol. 25 (2), 305-314.

DOI: $10.1007 / \mathrm{s} 11274-008-9892-2$

Czajkowski R., de Boer W. J., van Veen J. A. y van der Wolf J. M. (2012). Studies on the interaction between the biocontrol agent, Serratia plymuthica A30, and blackleg-causing Dickeya sp. (biovar 3) in potato (Solanum tuberosum). Plant Pathol. 61 (4), 677-688. DOI: 10.1111/j.1365-3059.2011.02565.x

Datta M., Palit R., Sengupta C., Pandit M. K. y Banerjee S. (2011). Plant growth promoting rhizobacteria enhance growth and yield of chilli (Capsicum annuum L.) under field conditions. AJCS 5 (5), 531-536.

De-Bashan L. E., Holguin G., Glick B. R. y Bashan Y. (2007). Bacterias promotoras de crecimiento en plantas para propósitos agrícolas y ambientales. En: Microbiología agrícola (R. Ferrara- Cerrato y A. Alarcón, Eds.). Editorial Trillas, México, D.F., México, pp. 170-209.

De Vleesschauwer D. y Hofte M. (2007). Using Serratia plymuthica to control fungal pathogens of plants. CAB Rev. Perspect. Agric. Vet. Sci. Nutr. Nat. Resour. 2 (46), 1-12.

DOI: $10.1079 /$ PAVSNNR20072046

Dias A. C. F., Costa F. E. C., Andreote F. D., Lacava P. T., Teixeira M. A., Assumpcao L. C., Araujo W. L., Azevedo J. L. y Melo I. S. (2009). Isolation of micropropagated strawberry endophytic bacteria and assessment of their potential for plant growth promotion. World J. Microbiol. Biotechnol. 25 (2), 189-195.

DOI: $10.1007 / \mathrm{s} 11274-008-9878-0$
FAOSTAT (2013). División estadística. Food and Agriculture Organization of the United Nations [en línea]. http://faostat3.fao.org/browse/Q/QC/S 10/11/2016

Fitriatin B. N., Yuniarti A., Turmuktini T. y Ruswandi F. K. (2014). The effect of phosphate solubilizing microbe producing growth regulators on soil phosphate, growth and yield of maize and fertilizer efficiency on Ultisol. Eurasian J. Soil Sci. 3 (2), 101-107.

DOI: 10.18393 /ejss.34313

Frank J. A., Reich C. I., Sharma S., Weisbaum J. S., Wilson B. A. y Olsen G. J. (2008). Critical evaluation of two primers commonly used for amplification of bacterial 16S rRNA genes. Appl. Environ. Microbiol. 74 (8), 2461-2470. DOI: 10.1128/AEM.02272-07

Galtier N., Gouy M. y Gautier C. (1996). SEA VIEW and PHYLO_WIN: two graphic tools for sequence alignment and molecular phylogeny. Comput. Appl. Biosci. 12 (6), 543-548.

DOI: 10.1093/bioinformatics/12.6.543

García M. C., Taboada G. O. R., López S. H. López P. A., Mora A. G. y Tlapal B. B. (2011). Calidad de plántulas de Chile 'Poblano' en la Sierra Nevada de Puebla, México. Rev. Fitotec. Mex. 34 (2), 115-121.

González P. E., Yáñez M. M. J., Santiago S. V. y Montero P. A. (2004). Biodiversidad fungosa en la marchitez del chile y algunos factores involucrados en Tlacotepec de José Manzo, El Verde, Puebla. Agrociencia 38 (6), 653-661.

Gravel V., Dorais M. y Ménard C. (2012). Organic fertilization and its effect on development of sweet pepper transplants. HortScience 47 (2), 198-204.

Guzmán M. y Sánchez A. (2003). Influence of nitrate and calcium increments on development, growth and early yield in sweet pepper plants. Acta Horticulturae 609, 207-211. DOI: 10.17660/ActaHortic.2003.609.29

Hahm M. S., Sumayo M., Hwang Y. J., Jeon S. A., Park S. J., Lee J. Y., Ahn J. H., Kim B. S., Ryu C. M. y Ghim S. Y. (2012). Biological control and plant growth promoting capacity of rhizobacteria on pepper under greenhouse and field conditions. J. Microbiol. 50 (3), 380-385. DOI: 10.1007/s12275-012-1477-y

Hall T. A. (1999). BioEdit: a user-friendly biological sequence alignment editor and analysis program for Windows 95/98/NT. Nucl. Acids Symp. Ser. 41, 95-98.

Hariprasad P. y Niranjana S. R. 2009. Isolation and characterization of phosphate solubilizing rhizobacteria to improve plant health of tomato. Plant Soil 316 (1), 13-24. DOI: 10.1007/s11104-008-9754-6

Kim Y. C., Kim K. Y., Jung H. y Park S. K. (2008). An effective biocontrol bioformulation agains Phytophthora blight of pepper using growth mixtures of combined chitinolytic bacteria under different field conditions. Eur. J. Plant Pathol. 120 (4), 373-382. DOI: 10.1007/ s10658-007-9227-4 
Khan M. S., Zaidi A, Ahemad M., Oves M. y Wani P. A. (2010). Plant growth promotion by phosphate solubilizing fungi - current perspective. Arch. Agron. Soil Sci. 56 (1), 73-98. DOI: 10.1080/03650340902806469

Luna M. L., Martínez P. R. A., Hernández I. M., Arvizu M. S. M. y Pacheco A. J. R. (2013). Caracterización de rizobacterias aisladas de tomate y su efecto en el crecimiento de tomate y pimiento. Rev. Fitotec. Mex. $36(1), 63-69$.

Manas R. S., Ramesh C. R. y Lata (2010). Tomato crop: plant growth promoting rhizobacteria in growth, yield and productivity. En: Tomatoes: Agricultural procedures, pathogen interactions and health effects (E. D. Aube y F. H. Poole, Eds.), Nova Science Publishers, Nueva York, EUA, pp. 147-159.

McCullagh M., Utkhede R., Menzies J. G., Punja Z. K. y Paulitz T. C. (1996). Evaluation of plant growthpromoting rhizobacteria for biological control of Pythium root rot of cucumbers grown in rockwool and effects on yield. Eur. J. Plant Pathol. 102 (8), 747-755. DOI: 10.1007/BF01877149

Morgulis A., Coulouris G., Raytselis Y., Madden T. L., Agarwala R. y Schäffer A. A. (2008). Database indexing for production MegaBLAST searches. Bioinformatics 24 (16), 1757-1764.

DOI: $10.1093 /$ bioinformatics/btn322

Muller H., Westendorf C., Leitner E., Chernin L., Riedel K., Schmidt S., Eberl L. y Berg G. (2009). Quorumsensing effects in the antagonistic rhizosphere bacterium Serratia plymuthica HRO-C48. FEMS Microbiol. Ecol. 67 (3), 468-478.

DOI: $10.1111 / j .1574-6941.2008 .00635 . x$

Pan B., Bai Y. M., Leibovitch S. y Smith D. L. (1999). Plant-growth-promoting rhizobacteria and kinetin as ways to promote corn growth and yield in a shortgrowing-season area. Eur. J. Agron. 11 (3-4), 179-186. DOI: 10.1016/S1161-0301(99)00029-5

Pan B., Vessey J. K. y Smith D. L. (2002). Response of field-grown soybean to co-inoculation with the plant growth promoting rhizobacteria Serratia proteamaculans or Serratia liquefaciens, and Bradyrhizobium japonicum pre-incubated with genistein. Eur. J. Agron. 17 (2), 143-153.

DOI: 10.1016/S1161-0301(01)00148-4

Pikovskaya R. I. (1948). Mobilization of phosphorus in soil in connection with the vital activity of some microbial species. Mikrobiologiya 17, 362-370.

Ponmurugan P. y Gopi C. (2006). In vitro production of growth regulators and phosphatase activity by phosphate solubilizing bacteria. Afr. J. Biotechnol. 5 (4), 348-350.

Puerta A. C. E., Russian L. T. y Ruiz S. C. A. (2012). Producción de plántulas de pimentón (Capsicum annuum
L.) en sustratos orgánicos a base de mezclas con fibra de coco. UDO Agrícola 12 (2), 298-306.

Pulawska J., Willems A., De Meyer S. E. y Süle S. (2012). Rhizobium nepotum sp. nov. isolated from tumors on different plant species. Syst. Appl. Microbiol. 35 (4), 215-220. DOI: 10.1016/j.syapm.2012.03.001

Reyes-Ramírez A., López-Arcos M., Ruiz-Sánchez E., Latournerie-Moreno L., Pérez-Gutiérrez A., LozanoContreras M. G. y Zavala-León M. J. (2014). Efectividad de inoculantes microbianos en el crecimiento y productividad de chile habanero (Capsicum chinense Jacq.). Agrociencia 48 (3), 285-294.

Rosselló-Mora R. y Amann R. (2001). The species concept for prokaryotes. FEMS Microbiol. Rev. 25 (1), 39-67. DOI: $10.1111 /$ j.1574-6976.2001.tb00571.x

Russo V. M. (2004). Greenhouse-grown transplants as an alternative to bare-root transplants for onion. HortScience 39 (6), 1267-1271.

Russo V. M. y Perkins-Veazie P. (2010). Yield and nutrient content of bell pepper pods from plants developed from seedlings inoculated, or not, with microorganisms. HortScience 45 (3), 352-358.

Salazar-Jara F. I. y Juárez-López P. (2013). Requerimiento macronutrimental en plantas de chile (Capsicum annuum L.). Biociencias 2 (2), 27-34.

Sandhya V., Ali S. Z., Venkateswarlu B., Reddy G. y Grover M. (2010). Effect of osmotic stress on plant growth promoting Pseudomonas spp. Arch. Microbiol. 192 (10), 867-876. DOI: 10.1007/s00203-010-0613-5

Saraf M., Kumar J. C. y Patel D. (2011). The role of ACC deaminase producing PGPR in sustainable agriculture. En: Plant growth and health promoting bacteria (D. K. Maheshwari, Ed.). Springer Berlin Heidelberg, Microbiology Monographs Vol. 18, pp. 365-385.

DOI: 10.1007/978-3-642-13612-2_16

Sarathambal C., Ilamurugu K., Balachandar D., Chinnadurai C. y Gharde Y. (2015). Characterization and crop production efficiency of diazotrophic isolates from the rhizosphere of semi-arid tropical grasses of India. Appl. Soil Ecol. 87, 1-10. DOI: 10.1016/j.apsoil.2014.11.004

SAS (2002). Software: the SAS System for Windows version 9.0. Statistical Analysis System Institute Inc., Cary, NC 25513, Carolina del Norte, EUA.

Son H. J., Park G. T., Cha M. S. y Heo M. S. (2006). Solubilization of insoluble inorganic phosphates by a novel salt- and $\mathrm{pH}$ tolerant Pantoea agglomerans R-42 isolated from soybean rhizosphere. Bioresour. Technol. 97 (2), 204-210. DOI: 10.1016/j.biortech.2005.02.021 Sharma A., Pathak A., Sahgal M., Meyer J. M., Wray V. y Johri B. N. (2007). Molecular characterization of plant growth promoting rhizobacteria that enhance peroxidase and phenylalanine ammonia-lyase activities in chile (Capsicum annuum L.) and tomato (Lycopersicon 
esculentum Mill.). Arch. Microbiol. 188 (5), 483-494. DOI: $10.1007 / \mathrm{s} 00203-007-0270-5$

Shen S. S., Park C. S. y Kim W. (2006). Effect of hydrogel on survial of Serratia plymuthica A21-4 in soils and plant disease suppression. Plant Pathol. J. 22 (4), 364368. DOI: 10.5423/PPJ.2006.22.4.364

Smith V. H. y Schindler V. W. (2009). Eutrophication science: Where do we go from here? Trends Ecol. Evol. 24 (4), 201-207. DOI: 10.1016/j.tree.2008.11.009

Tamura K., Stecher G., Peterson D., Filipski A. y Kumar S. (2013). MEGA6: Molecular evolutionary genetics analysis version 6.0. Mol. Biol. Evol. 30 (12), 27252729. DOI: $10.1093 / \mathrm{molbev} / \mathrm{mst} 197$
Weyer P. J., Cerhan J. R., Kross B. C., Hallberg G. R., Kantamneni J., Breuer G., Jones M. P., Zheng W. y Lynch C. F. (2001). Municipal drinking water nitrate level and cancer risk in older women: the Iowa women's health study. Epidemiology 12 (3), 327-338. DOI: 10.1097/00001648-200105000-00013

Yau J. A., Diánez F., Marín F., Carretero F. y Santos M. (2013). Screening and evaluation of potential biocontrol fungi and bacteria foliar endophytes against Phytophthora capsici and Phytophthora parasitica on pepper. J. Food Agric. Environ. 11 (2), 490-495. 